\title{
Calibration Method for the Gas-Chromatographic Retention Time of Polychlorinated Biphenyl Congeners
}

\author{
Hiroshi Kato, ${ }^{*}$ Yoshihisa Ueda, $* *$ and Munetaka NAKATA*** \\ *System Development Division, JEOL Ltd., Musashino 3-1-2, Akishima, Tokyo 196-8558, Japan \\ **Analytical Instrument Division, JEOL Ltd., Musashino 3-1-2, Akishima, Tokyo 196-8558, Japan \\ ***Graduate School of Bio-Applications and Systems Engineering, Tokyo University of Agriculture and Technology, \\ Naka-cho 2-24-16, Koganei, Tokyo 184-8588, Japan
}

\begin{abstract}
A calibration method for the gas-chromatographic (GC) retention time of polychlorinated biphenyl (PCB) congeners is presented. This method calculates retention time of all PCB congeners in the GC system of an analytical laboratory from one reported in the literature with a commercial PCB mixture and some pure congener standards. Two corrections were implemented to compensate for any retention differences arising from the GC condition changes, such as the column dimensions, temperature programs and flow rates. One is an instrument function correction that compensates for common congener behavior. The other is a selectivity correction that compensates for individual congener behavior. For a selectivity correction, new descriptors of a quantitative structure-retention relationship (QSRR) were developed. The method was applied to several stationary phases to evaluate its ability and precision. The standard errors of the calculated retention times were in most cases within a chromatographic peak width.
\end{abstract}

(Received January 12, 2000; Accepted April 17, 2000)

Polychlorinated biphenyl (PCB) is one of the most widespread persistent organic pollutants. PCB theoretically consists of 209 congeners, of which around 150 have been reported at significant levels in the environment. Because environmental behavior of PCBs is congener-dependent, a comprehensive, quantitative and congener-specific (CQCS) analysis is highly demanded. ${ }^{1}$ The CQCS PCB analysis uses high-resolution gas chromatography (HRGC) with sensitive and selective detection by an electron capture detector (ECD) or a mass spectrometer (MS).

There are so many PCB congeners that the analysis of an acquired chromatogram is labor-intensive and time-consuming. Therefore, a fully automated quantitative analysis is desired in an analytical laboratory where many environmental samples are routinely quantified. The important process in the analysis is the identification of each congener in accordance with its retention time. Automated identification requires precise retention times of the GC system in an analytical laboratory, since all chromatographic peaks arising from congeners should be individually quantified when they elute separately.

Recently, Frame has reported a complete retention database on 27 HRGC systems using 20 stationary phases with all 209 pure congener standards commercially available. ${ }^{2}$ The retention database includes multiple data sets measured by different GC systems with the same stationary phases. In a comparison of these data sets, different parameters of column dimensions, film thickness, temperature programs and flow rates change the retention time substantially. The retention differences are mainly due to the common behavior of congeners, which exhibit smooth curvature differences along with an increase of the retention time. Additionally, small but significant differences due to individual behavior of congeners change the elution orders as well as the resolvability of some adjacent congeners. These retention differences suggest that congener identification should not be attempted solely by matching the values in the retention database. ${ }^{2}$

Retention indices (RI) are used to remove any influence of a specific GC condition. ${ }^{3-6}$ However, an RI calculation can not compensate for individual congener behavior that makes the elution orders change, because it is a linear conversion. All 209 congener standards are still expensive and cannot be utilized in all analytical laboratories. We have thus developed a new calibration method that compensates for retention changes between different GC systems with the same stationary phase. In conjunction with the valuable retention database reported by Frame, ${ }^{2}$ the proposed method can be used to predict the retention time of congeners whose standards are not available in an analytical laboratory.

\section{Experimental}

A commercial PCB mixture (Kanechlor) was purchased from GL Sciences Inc., Japan. A PCB standard mixture was obtained from Wellington Laboratories, USA. The standard mixture contained the first and last eluted congeners in each chlorine homologue at a DB-5 column.

Chromatograms of PCB samples were measured using a JMSGC mate mass spectrometer (JEOL Ltd., Japan) connected with a HP 6890 gas chromatograph (Hewlett-Packard, USA). The retention time was obtained in the following GC columns:

\section{SPB-1 (Supelco) : $30 \mathrm{~m} \times 0.25 \mathrm{~mm}, 0.25 \mu \mathrm{m}$ $100 \%$ dimethy siloxane}

DB-5 (J\&W) $\quad: 60 \mathrm{~m} \times 0.25 \mathrm{~mm}, 0.25 \mu \mathrm{m}$ $5 \%$ diphenyl dimethy siloxane 


\author{
DB-17 (J\&W) : $30 \mathrm{~m} \times 0.25 \mathrm{~mm}, 0.25 \mu \mathrm{m}$ \\ $50 \%$ diphenyl dimethyl siloxane \\ HT-8 (SGE) $\quad: 50 \mathrm{~m} \times 0.22 \mathrm{~mm}, 0.25 \mu \mathrm{m}$ \\ 1,7dicarba-closo-dodecarborane \\ phenymethyl siloxiane
}

Samples were injected with the pulsed splitless mode. Helium was used as the carrier gas with a flow rate of $1 \mathrm{ml} / \mathrm{min}$, except for the HT- 8 column, which was $0.8 \mathrm{ml} / \mathrm{min}$. The oventemperature programs used are described in the results and discussion section. The temperatures of injection and detection were $300^{\circ} \mathrm{C}$ and $280^{\circ} \mathrm{C}$, respectively. MS was operated in the selected ion monitoring (SIM) mode at two abundant masses of each homologue. The reproducibility of the retention time under the same GC conditions was less than $10 \%$ of the chromatographic peak width at half height.

Computer programs were developed by Visual Basic compiler (Microsoft, Japan) to implement the calibration method described in the next section. The retention database reported by Frame ${ }^{2}$ was obtained as supplementary material from the world wide web home page of Fresenius's Journal. ${ }^{7}$ In this study, all retention data were manipulated in relative retention time (RRT) against the sum of the retention time of PCB 52 and $180 .{ }^{8}$

\section{Calibration Method}

\section{Instrument function correction}

The retention time of $\mathrm{PCB}$ congeners is dependent on the column dimensions (length, internal diameter), film thickness, temperature programs, carrier gas and flow rates. A set of these parameters characterizes a specific GC system, and is called an instrument function (IF). The retention difference therefore arises from the IF difference between two GC systems. If the IF difference is evaluated, the retention time in one GC system can be converted to that in another GC system. This relationship is expressed as

$$
r_{m_{i}}=F\left(r_{o_{i}}\right)+e_{f_{i}}
$$

In this equation, $r_{m_{i}}$ is the retention time of congener $i$ in the target GC system, $r_{o i}$ is the corresponding retention time in a reference GC system, $F$ is the IF difference between two GC systems, called a conversion function, and $e_{f_{i}}$ is an error not explained by the conversion function. Because the IF difference is expected to vary smoothly along with an increase in retention time, we employed a polynomial function to express the conversion function.

\section{Selectivity correction}

Although the IF correction can remove the common retention behavior of congeners, the contribution of individual congener behavior still remains in the residual errors $\left(e_{f_{i}}\right)$. We have found that the individual congener behavior can be explained as selectivity differences between GC systems. ${ }^{9}$ The selectivity differences can be evaluated by a mathematical model named quantitative structure-retention relationship (QSRR). ${ }^{10}$ This model explains the retention time based on structure-related parameters, so-called descriptors. The QSRR descriptors used were 20 chlorine substitution patterns of one phenyl ring, ${ }^{11,12}$ and are here called single ring substitution pattern (SRSP) descriptors.

The SRSP descriptors are implemented as so-called dummy variables of the regression analysis; each descriptor takes a value 0,1 or 2 , depending on whether such a pattern does not appear, appears once or appears twice, respectively. A leastsquares method was applied to obtain the coefficients of the SRSP descriptors. Each coefficient represents the selectivity contribution of a corresponding SRSP descriptor. The equation of the regression analysis can be expressed as

$$
e_{f_{i}}=\sum_{j} D_{s j i} x_{j}+e_{s_{i}}
$$

where $D_{s j i}$ is the $j$-th dummy variable of congener $i, x_{j}$ is the SRSP coefficient and $e_{s i}$ is the residual error of the regression analysis. A set of SRSP coefficients is called a selectivity profile.

\section{Overall calibration}

In most cases, residual errors $\left(e_{s_{i}}\right)$ of the selectivity correction show a systematic curvature along with $r_{o i}$, since $e_{s_{i}}$ are much smaller than $e_{f_{i}}$ so that the selectivity correction reveals a small IF difference not removed by the first attempt of the IF correction. To express the curvature, we employed a cubic smoothing spline, which has been widely used to express this kind of curvature exhibited with a large number of data points. ${ }^{13-15}$ The curve obtained from the $e_{s i}$ is added to the conversion function. Then, the residual errors $\left(e_{i}\right)$ of the conversion function are re-calculated according to

$$
e_{f_{i}}=r_{m_{i}}-\left\{F_{1}\left(r_{o_{i}}\right)+F_{2}\left(r_{o_{i}}\right)\right\}
$$

where $F_{1}$ and $F_{2}$ are conversion functions derived by the first and second attempts, respectively. The regression analysis of Eq. (2) is again applied to the new residual errors $\left(e_{f_{i}}\right)$. This procedure is repeated until no systematic errors are observed in the $e_{s i}$. Thus, the overall calibration model is defined as

$$
r_{m_{i}}=\sum_{k} F_{k}\left(r_{o_{i}}\right)+\sum_{j} D_{s_{j i}} x_{j}+e_{s_{i}} .
$$

In this equation, the first term of the right-hand side represents the IF correction and the second term represents the selectivity correction.

\section{Extracted selectivity profile pattern descriptor}

There are 27 data sets in the retention database. ${ }^{2}$ For a comparison of the selectivity differences among these data sets, the calibration method described above has been applied to obtain 26 selectivity profiles in reference to GC system 3 of the dimethylsiloxane stationary phase, which is used as the leastpolar stationary phase. With a careful examination of these selectivity profiles, we have found that the selectivity profiles due to the GC condition differences exhibited similar patterns to those due to the stationary phase differences. The similarity suggests that the patterns of the selectivity profiles arising from the GC condition differences are hidden in the retention database. To extract such patterns, we employ a principalcomponent analysis (PCA). ${ }^{16,17}$

Each selectivity profile derived from the retention database is divided by the standard deviation of the calibration result in order that PCA analyzes selectivity profile data with the same precision. A matrix $\mathbf{X}$ is constructed with 26 row vectors $\left(\mathbf{x}^{\prime}\right)$ of the corrected selectivity profiles. PCA is applied to decompose $\mathbf{X}$ into a score matrix $(\mathbf{T})$, a loading matrix $(\mathbf{P})$ and an error matrix (E),

$$
\mathbf{X}=\mathbf{T} \mathbf{P}^{\prime}+\mathbf{E},
$$


Table 1 GC systems in the retention database ${ }^{2}$ used for the calibration

\begin{tabular}{|c|c|c|c|c|c|c|c|c|c|c|c|c|}
\hline & \multirow[b]{2}{*}{$\mathrm{GC}_{\text {system }}^{\mathrm{a}}$} & \multicolumn{3}{|c|}{ Column } & \multicolumn{6}{|c|}{ Temperature program } & \multicolumn{2}{|c|}{ Chromatogram } \\
\hline & & $\begin{array}{l}\text { Length/ } \\
\mathrm{m}\end{array}$ & $\begin{array}{c}\text { I.D./ } \\
\text { mm }\end{array}$ & $\begin{array}{l}\text { Film/ } \\
\mu \mathrm{m}\end{array}$ & $\begin{array}{l}\text { Initial } \\
\text { temp./ } \\
{ }^{\circ} \mathrm{C}\end{array}$ & $\begin{array}{l}\text { Initial } \\
\text { hold/ } \\
{ }^{\circ} \mathrm{C}\end{array}$ & $\begin{array}{c}1 \mathrm{st} \\
\text { rate/ } \\
{ }^{\circ} \mathrm{C} \min ^{-1}\end{array}$ & $\begin{array}{c}1 \mathrm{st} \\
\text { break/ } \\
{ }^{\circ} \mathrm{C}\end{array}$ & $\begin{array}{c}\text { 2nd } \\
\text { rate/ } \\
{ }^{\circ} \mathrm{C} \mathrm{min}{ }^{-1}\end{array}$ & $\begin{array}{l}\text { Final } \\
\text { temp./ } \\
{ }^{\circ} \mathrm{C}\end{array}$ & $\begin{array}{c}\mathrm{RT}^{\mathrm{b}} \\
52+180 / \\
\min \end{array}$ & $\begin{array}{c}\mathrm{W} @ 1 / 2 \mathrm{H}^{\mathrm{c}} / \\
\min \end{array}$ \\
\hline 1 & DB-1 & 30 & 0.25 & 0.25 & 90 & 2 & 15 & 165 & 2.5 & 255 & 44.17 & 0.060 \\
\hline 2 & DB-1 & 30 & 0.25 & 0.25 & 75 & 2 & 15 & 150 & 2.5 & 260 & 59.69 & 0.064 \\
\hline 3 & RTX-1 & 60 & 0.25 & 0.25 & 75 & 2 & 15 & 150 & 1.5 & 285 & 106.73 & 0.084 \\
\hline 4 & SPB-Octyl & 30 & 0.25 & 0.25 & 90 & 2 & 15 & 165 & 3 & 280 & 54.97 & 0.053 \\
\hline 5 & SPB-Octyl & 30 & 0.25 & 0.25 & 75 & 2 & 15 & 150 & 3 & 270 & 63.71 & 0.053 \\
\hline 6 & SPB-Octyl & 60 & 0.25 & 0.25 & 75 & 2 & 15 & 150 & 1.2 & 270 & 119.83 & 0.135 \\
\hline 7 & SPB-Octyl & 30 & 0.25 & 0.25 & 75 & 2 & 15 & 150 & 2.5 & 280 & 75.19 & 0.077 \\
\hline 8 & CP-Sil5-C18 & 100 & 0.25 & 0.10 & 75 & 2 & 15 & 150 & 0.75 & 268 & 192.54 & 0.200 \\
\hline 9 & CP-Sil5-C18 & 100 & 0.32 & 0.10 & 75 & 2 & 15 & 150 & 0.75 & 250 & 161.60 & 0.195 \\
\hline 11 & RTX-5 & 60 & 0.25 & 0.25 & 75 & 2 & 15 & 150 & 1.2 & 300 & 128.43 & 0.118 \\
\hline 16 & DB-17 & 30 & 0.25 & 0.25 & 100 & 1 & 3 & ${ }^{\mathrm{d}}$ & $-^{\mathrm{d}}$ & 290 & 85.66 & 0.066 \\
\hline 22 & HT-8 & 50 & 0.22 & 0.25 & 80 & 2 & 30 & 170 & 3 & 320 & 76.55 & 0.090 \\
\hline 23 & CNBP & 25 & 0.25 & 0.25 & 75 & 5 & 4 & 155 & 2 & 250 & 97.70 & 0.125 \\
\hline 26 & CNBP & 30 & 0.32 & 0.25 & 80 & 1 & 2.5 & $-\mathrm{d}$ & $-\mathrm{d}$ & 270 & 114.45 & 0.090 \\
\hline
\end{tabular}

a. GC system number denoted in the original paper. b. Sum of the retention time of PCB 52 and 180. c. Mean of the chromatographic peak widths at the half height of PCB 52 and 180. d. One-step temperature program.

where the prime implies a transposed matrix. PCA extracts the relevant information in $\mathbf{X}$ into the first few principal components. In this application, vector $\mathbf{p}_{\mathbf{1}}$ of the first principal component in the loading matrix (P) represents a major selectivity profile governed in $\mathbf{X}$, the second vector $\left(\mathbf{p}_{2}\right)$ represents a next major profile not explained by $\mathbf{p}_{\mathbf{1}}$, and so on.

Using vectors and matrices, the selectivity correction of Eq. (2) is expressed with an error vector $\left(\mathbf{e}_{\mathbf{f}}\right)$ of the IF correction, a SRSP descriptor matrix $\left(\mathbf{D}_{\mathbf{s}}\right)$, a selectivity profile vector $(\mathbf{x})$ and an error vector $\left(\mathbf{e}_{\mathrm{s}}\right)$ of the selectivity correction,

$$
\mathbf{e}_{\mathrm{f}}=\mathbf{D}_{\mathrm{s}} \mathbf{x}+\mathbf{e}_{\mathrm{s}}
$$

The selectivity profile vector $(\mathbf{x})$ can be expressed by a corresponding score vector $(\mathbf{t})$ and the loading matrix $(\mathbf{P})$,

$$
\mathbf{x}^{\prime}=\mathbf{t}^{\prime} \mathbf{P}^{\prime}+\mathbf{e}^{\prime}
$$

Combining Eqs. (6) and (7) gives

$$
\mathbf{e}_{\mathrm{f}}=\mathbf{D}_{\mathrm{s}} \mathbf{P t}+\mathbf{e}_{\mathrm{s}}
$$

where an error vector in Eq. (7) is included in $\mathbf{e}_{\mathbf{s}}$. Introducing a new matrix $\left(\mathbf{D}_{\mathbf{e}}\right)$ from the product of $\mathbf{D}_{\mathbf{s}}$ and $\mathbf{P}$, we have

$$
\mathbf{e}_{\mathrm{f}}=\mathbf{D}_{\mathrm{e}} \mathbf{t}+\mathbf{e}_{\mathrm{s}}
$$

Because both $\mathbf{D}_{\mathbf{s}}$ and $\mathbf{P}$ are known, matrix $\mathbf{D}_{\mathbf{e}}$ is uniquely determined. Now, the regression coefficients determined in the selectivity correction are elements of $\mathbf{t}$ instead of $\mathbf{x}$. Equation (4) of the calibration model can be expressed using the new descriptor $\left(\mathbf{D}_{\mathbf{e}}\right)$ :

$$
r_{m_{i}}=\sum_{k} F_{k}\left(r_{o_{i}}\right)+\sum_{j} D_{e_{j i}} t_{j}+e_{s_{i}}
$$

The number of new descriptors corresponds to the effective number of the principal components. The new descriptors are called extracted selectivity profile pattern (ESPP) descriptors in contrast with the SRSP descriptors.
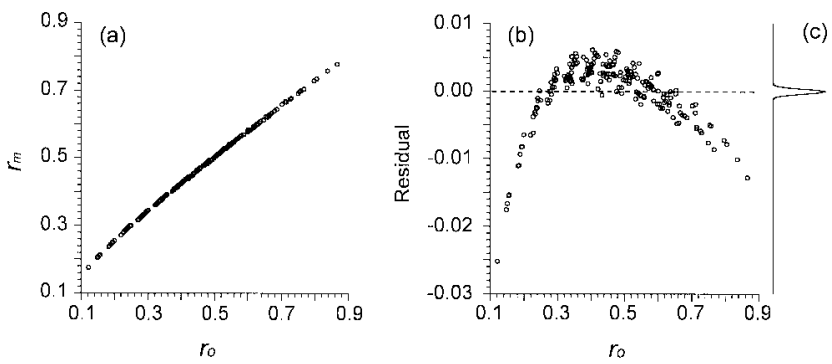

Fig. 1 (a) Target RRT $r_{m}$ (GC system 5) versus reference RRT $r_{o}$ (GC system 6). (b) Residual-error plot of the simple linear correction. (c) Gaussian peak with the corresponding width in Table 1.

\section{Results and Discussion}

The retention database reported by Frame $^{2}$ contains multiple data sets measured by different GC systems with the same stationary phase: three data sets of dimethylsiloxane (DB-1), four of $n$-octylmethysiloxane (SPB-Octyl), two of $n$ octadecylmethysiloxiane (CP-Sil5-C18) and two of $p$-cyano, $p^{\prime}$ allyloxy biphenylmethylsiloxane (CNBP) stationary phases. These retention data sets are good applications for the proposed calibration method to evaluate its ability and precision. The GC systems used are listed in Table 1.

An example of the calibration is demonstrated with the target retention $\left(r_{m_{i}}\right)$ of GC system 5 calibrated from the reference retention $\left(r_{o_{i}}\right)$ of GC system 6 with SPB-Octyl columns. As shown in Fig. 1, although the relation between the $r_{m i}$ and the $r_{o i}$ is strongly correlated, a non-linear contribution is revealed by a simple linear correction. A cubic function is applied for the first IF correction, of which the residual errors $\left(e_{f_{i}}\right)$ are shown in Fig. 2a. The order of the polynomial depends on the IF difference between two GC systems. The first attempt need not remove all contributions of the IF difference, because the correction is repeated, but should remove any major contributions of the IF difference; otherwise, the next selectivity correction would not work properly. The order of the polynomial was determined by a trial-and-error procedure. For the retention data used in this 


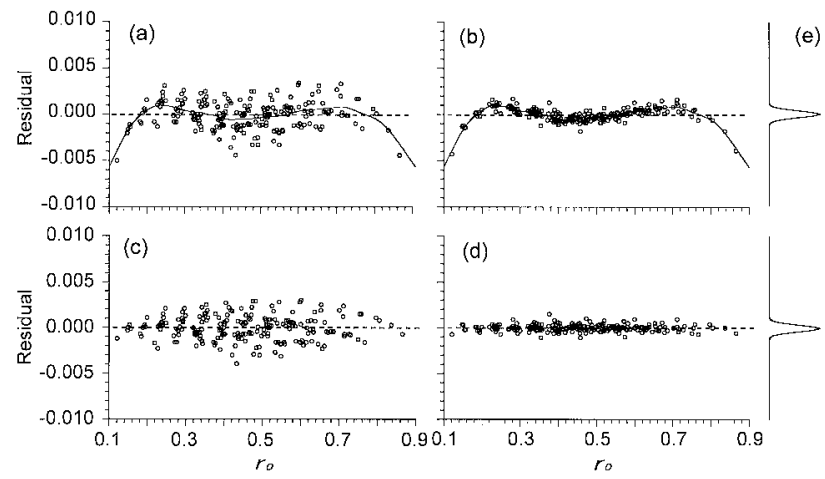

Fig. 2 Example of the calibration method applied to the retention data sets shown in Fig. 1. (a) Residual-error plot of the first IF correction. (b) Residual-error plot of the first selectivity correction. (c) Residual-error plot of the second IF correction. (d) Residual-error plot of the second selectivity correction. (e) Gaussian peaks with the corresponding width in Table 1.

study, the maximum fourth-order polynomials produced good results.

The selectivity correction is applied to the IF correction errors $\left(e_{f_{i}}\right)$ in Fig. 2a. The residual errors $\left(e_{s_{i}}\right)$ shown in Fig. 2b exhibit a systematic curvature due to an IF difference not removed by the first IF correction. The smoothing spline with a smoothing factor of $10^{-5}$ is applied to express the curvature. The solid lines in Figs. $2 \mathrm{a}$ and $2 \mathrm{~b}$ represent the extracted curve. The smoothing factor was highly dependent on the shape of curvature. When a small value is applied to express a complicated shape of curvature, a waved curve was sometimes generated to exhibit small changes in the data distribution. Therefore, the value of the factor was determined by a trial-and-error procedure. In this study, values ranging between $10^{-4}$ to $10^{-5}$ produced good results.

A second attempt is performed, and its results concerning $e_{f_{i}}$ and $e_{s_{i}}$ are shown in Figs. 2c and 2d, respectively. Because no systematic curvature is observed in the $e_{s i}$, the calculation is terminated. Otherwise, a third attempt would be necessary. Regarding the retention data in this study, a maximum of three iterations were sufficient to remove any systematic errors observed in the residual errors of the selectivity correction.

Using the retention data of all 209 congeners and the SRSP descriptors is the best condition for the calibration method. Calibrations under this condition were applied to the pairs of data listed in Table 1. The data set with a longer retention time of PCB 209 was used as reference retention, and another data set was calibrated as target retention. The calibration errors were evaluated as a root-mean-squared residual error, which is also called a standard error. For a comparison among different pairs of data sets, we calculated the relative root-mean-squared error (RRMSE) to the chromatographic peak width of the half height (W@1/2H), which is a criterion for an acceptable error of the calibration. Figure 3 shows the calibration results of the linear, IF and selectivity corrections to exhibit a compensated amount at each correction stage of the calibration.

It was found that the IF correction removes any non-linear contribution governed in the retention differences and produces much smaller RRMSEs than does the linear correction; also the selectivity correction accomplishes a further reduction of the RRMSEs. Error reductions of the IF and the selectivity correction are dependent on the data sets; no relation between them has been found. For example, a calibration between GC systems 6 and 4 shows a small effect of the IF correction and a

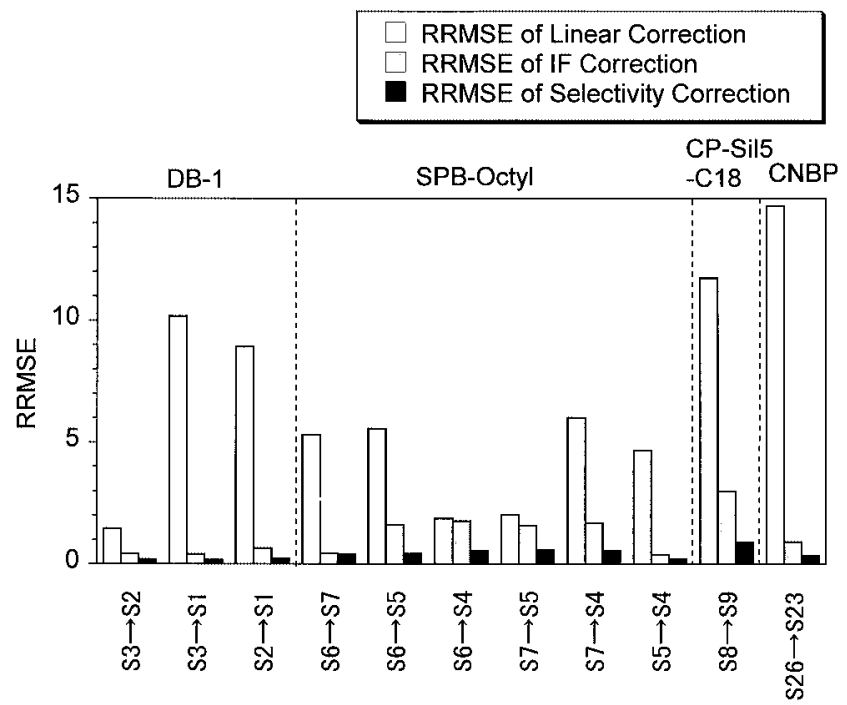

Fig. 3 RRMSEs of the calibrations applied to the data sets in the retention database. "SX $\rightarrow S Y$ " indicates that the retention data of GC system Y are calibrated from GC system X.

large effect of the selectivity correction. On the other hand, the calibration between GC systems 6 and 7 indicates a large effect of the IF correction, but a small effect of the selectivity correction. In spite of such differences, the final calibration errors are comparable to each other.

The RRMSEs of the selectivity correction, which are the final errors of the calibration, are also shown in Fig. 4, along with the results of other calibration conditions (described below). The final calibration errors are dependent on the stationary phases. The calibration of the DB-1 columns shows excellent results. The RRMSEs are less than a quarter of W@1/2H, and the residual errors are almost located within half of W@1/2H. The RRMSEs of the SPB-Octyl columns are two times larger than the DB-1 columns, except for the calibration between GC systems 4 and 5 , where the result is comparable to those of the DB- 1 columns. The CNBP columns show a large RRMSE of the linear correction, since the temperature programs are quite different between the GC systems; GC system 26 was measured in a one-step temperature program, but GC system 23 was measured in a two-step temperature program, and was additionally held for $10 \mathrm{~min}$ at $211^{\circ} \mathrm{C} .^{2}$ Nevertheless, the final result is comparable to those of the SPB-Octyl columns. The CP-Sil15-C18 columns show the worst result among the evaluated columns, but the selectivity correction works effectively to reduce any calibration errors three-times smaller than the IF correction does, and the final errors becomes less than W@1/2H.

We newly developed the ESPP descriptors from 27 data sets of the retention database. PCA of the dimension-reduction technique was applied to extract major selectivity profiles in the retention database. The major selectivity profiles were extracted in loading vectors of the transformed loading matrix (P). The loading vectors of the first four principal components are given in Table 2, in which the contribution rate of each principal component is also shown to represent how much variance of the original selectivity data is explained by the corresponding principal component. The ESPP descriptors are products of the SRSP descriptor matrix $\left(\mathbf{D}_{\mathbf{s}}\right)$ and the loading matrix $(\mathbf{P})$. The calibrations with ESPP descriptors were applied to the same data sets mentioned above to estimate the 
Table 2 Loading vectors of the first four principal components

\begin{tabular}{lrrrr}
\hline SRSP & PC-1 & PC-2 & PC-3 & PC-4 \\
\hline $0-$ & 0.088 & -0.142 & -0.154 & -0.545 \\
$2-$ & -0.169 & 0.008 & -0.011 & -0.364 \\
$3-$ & 0.159 & 0.031 & 0.135 & -0.082 \\
$4-$ & 0.189 & 0.215 & -0.290 & 0.330 \\
$2,6-$ & -0.398 & 0.067 & 0.103 & -0.094 \\
$2,5-$ & -0.038 & -0.072 & 0.282 & 0.317 \\
$2,4-$ & 0.052 & -0.035 & -0.320 & 0.211 \\
$2,3-$ & -0.228 & 0.305 & 0.075 & -0.151 \\
$3,5-$ & 0.418 & -0.238 & 0.353 & 0.015 \\
$3,4-$ & 0.241 & 0.358 & -0.021 & 0.063 \\
$2,4,6-$ & -0.014 & -0.452 & -0.396 & 0.094 \\
$2,3,6-$ & -0.398 & 0.100 & 0.273 & 0.242 \\
$2,3,5-$ & 0.087 & -0.206 & 0.245 & 0.126 \\
$2,4,5-$ & 0.167 & -0.156 & -0.086 & 0.147 \\
$2,3,4-$ & -0.101 & 0.428 & -0.231 & 0.141 \\
$3,4,5-$ & 0.379 & 0.231 & 0.196 & -0.229 \\
$2,3,5,6-$ & -0.232 & -0.248 & 0.271 & 0.049 \\
$2,3,4,6-$ & -0.132 & -0.220 & -0.240 & 0.072 \\
$2,3,4,5-$ & 0.098 & 0.099 & -0.069 & -0.141 \\
$2,3,4,5,6-$ & -0.162 & -0.086 & -0.151 & -0.263 \\
C.R.(\%) & 61.3 & 36.2 & 1.3 & 0.6 \\
\hline
\end{tabular}

a. Contribution rate.

ability of the selectivity correction. The ESPP descriptors derived from the four principal components and a constant term were used in a regression analysis of the selectivity correction. Increases of the number of ESPP descriptors with further principal components made no improvement. The RRMSEs of the calibration with ESPP descriptors are shown in Fig. 4, and are slightly larger than those of the optimal condition with SRSP descriptors. The final errors increase in the range between $2 \%$ and $28 \%$, depending on the pairs of data sets.

In an actual calibration procedure, standard congeners are required to determine the conversion function and the selectivity profile of the model equation. We employed an inexpensive commercial PCB mixture as a standard sample. Congener concentrations in the mixture have been precisely measured by Frame. ${ }^{18-20}$ Congeners are categorized into three groups with respect to their concentrations; congeners with more than 1.0 weight percent are called major, congeners with a range between 1.0 and 0.05 are minor and congeners with less than 0.05 are trace or undetectable. There are 61 major congeners in a commercial mixture with a chlorine range between 2 and 8 , but there are no major congeners with chlorine of 1,9 nor 10 Therefore, we additionally employed a commercially available pure standard mixture. The standard mixture is a PCB window defining mixture. It contains 19 pure congeners that are the first and last eluted congeners of each homologue at the DB-5 column. Consequently, the total number of standard congeners is 78 , because two congeners are included in both samples. The same data sets mentioned above were used to evaluate calibrations with 78 congeners and ESPP descriptors. The calibration model was estimated from the retention of 78 congeners, and was then used to predict the retention of other congeners. The RRMSEs shown in Fig. 4 were calculated from the residual errors of all 209 congeners to compare with other condition results. A reduction of the number of congeners for the model estimation produced worse results than the other condition. The increases in the calibration errors are in the range between $6 \%$ and $26 \%$ compared to the condition of 209 congeners with the ESPP descriptors, and between $13 \%$ and

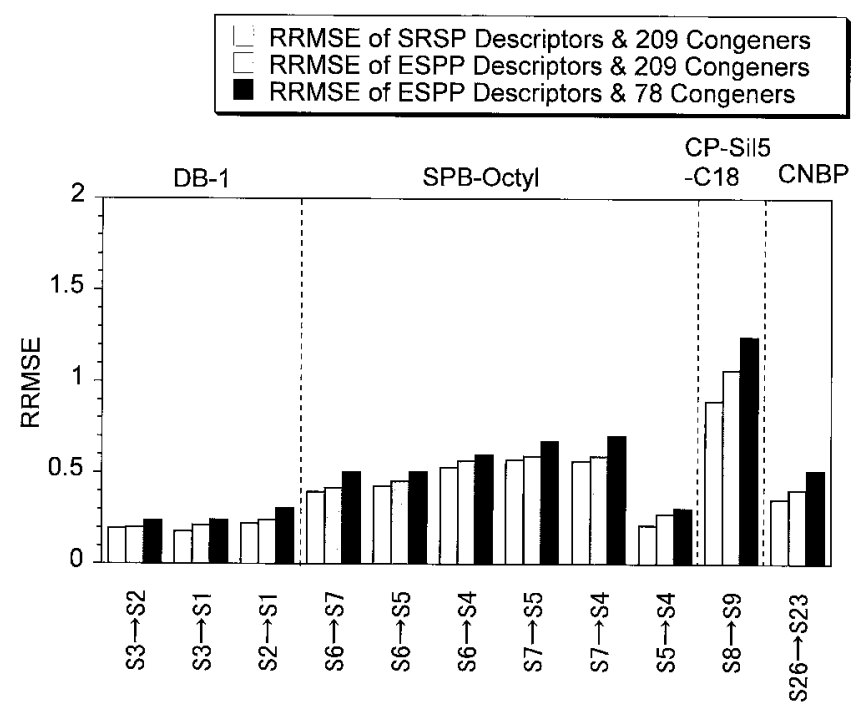

Fig. 4 RRMSE comparison under different calibration conditions. "SX $\rightarrow$ SY" indicates that the retention data of GC system Y are calibrated from GC system X.

$44 \%$ compared to the optimal condition of 209 congeners with the SRSP descriptors. Although the calibration model estimated with the ESPP descriptors and with the limited retention data enlarges the calibration errors, the increases are not significant compared with the total compensated amounts of the calibration.

The calibration method was applied to the retention data measured in our GC system with the SPB-1, DB-5, DB-17 and HT-8 columns. These columns have been proposed for singlecolumn and dual-column CQCS PCB analyses. ${ }^{1,21}$ The columns used had the same dimensions and film thickness as the corresponding columns used in the retention database. The retention time was obtained with the same temperature program in the retention database and the different conditions shown in Table 3.

Measured SIM chromatograms showed typical peak patterns due to the dominant major congeners, whose peaks were easily identified by referring to the literature. ${ }^{22}$ In addition to major congeners, minor congeners with strong intensities were also identified. The number of minor congeners varied between 40 and 50 due to the different resolvability of the GC columns. The retention data of the minor congeners were not included in the estimation of the calibration model, but were included in calculations of the RRMSEs for a validation purpose. The measured data were calibrated in reference to the data set of the corresponding GC system with the same stationary phase in the retention database. The calibration results are shown in Fig. 5, which represent the RRMSEs of the linear, IF and selectivity corrections.

In the DB-5 and DB-17 columns, the RRMESs of the linear correction are small in the GC methods with the same temperature programs of the retention database, and are large with different temperature programs. In the HT-8 column, however, the RRMSE with the same temperature program is larger than that of different temperature programs. Regardless of the linear-correction results, the IF correction compensated for any non-linear contribution, and reduced the RRMSEs remarkably. The calibration errors were further reduced by a selectivity correction. The final errors were comparable to 
Table 3 Temperature programs used for the calibration in this study

\begin{tabular}{|c|c|c|c|c|c|c|c|c|c|c|c|}
\hline & & & \multirow{2}{*}{$\begin{array}{l}\text { Reference }^{\mathrm{a}} \\
\text { GC system }\end{array}$} & \multirow{2}{*}{$\begin{array}{l}\text { Initial } \\
\text { temp./ } \\
{ }^{\circ} \mathrm{C}\end{array}$} & \multicolumn{4}{|c|}{ Temperature program } & \multirow{2}{*}{$\begin{array}{l}\text { Final } \\
\text { temp./ } \\
{ }^{\circ} \mathrm{C}\end{array}$} & \multicolumn{2}{|c|}{ Chromatogram } \\
\hline \multicolumn{3}{|c|}{ GC method } & & & Initial & 1st & $1 \mathrm{st}$ & 2nd & & $\begin{array}{c}\mathrm{RT}^{\mathrm{b}} \\
52+180\end{array}$ & $\mathrm{~W} @ 1 / 2 \mathrm{H}^{\mathrm{c}} /$ \\
\hline \multirow[t]{2}{*}{ SPB-1 } & 1 & $* \mathrm{~d}$ & 1 & 90 & 2 & 15 & 165 & 2.5 & 255 & 48.15 & 0.082 \\
\hline & 2 & $*$ & 2 & 75 & 2 & 15 & 150 & 2.5 & 260 & 57.89 & 0.101 \\
\hline \multirow[t]{2}{*}{ DB-5 } & 3 & $*$ & 11 & 75 & 2 & 15 & 150 & 1.2 & 300 & 125.57 & 0.119 \\
\hline & 4 & & 11 & 75 & 2 & 15 & 150 & 2.5 & 300 & 84.17 & 0.082 \\
\hline \multirow[t]{2}{*}{ DB-17 } & 5 & $*$ & 16 & 100 & 1 & 3 & $\underline{-}^{\mathrm{e}}$ & $-^{\mathrm{e}}$ & 290 & 87.52 & 0.089 \\
\hline & 6 & & 16 & 75 & 2 & 15 & 150 & 3 & 280 & 64.89 & 0.077 \\
\hline \multirow[t]{2}{*}{ HT-8 } & 7 & $*$ & 22 & 80 & 2 & 30 & 170 & 3 & 320 & 67.48 & 0.081 \\
\hline & 8 & & 22 & 80 & 2 & 30 & 170 & 4 & 320 & 57.94 & 0.076 \\
\hline
\end{tabular}

a. GC system number of the retention database. The corresponding retention data were used as a reference in the calibration. b. Sum of the retention time of PCB 52 and 180. c. Mean of the chromatographic peak widths at the half height of PCB 52 and 180 . d. "**" indicates that the temperature program is identical to the reference GC system. e. One-step temperature program.

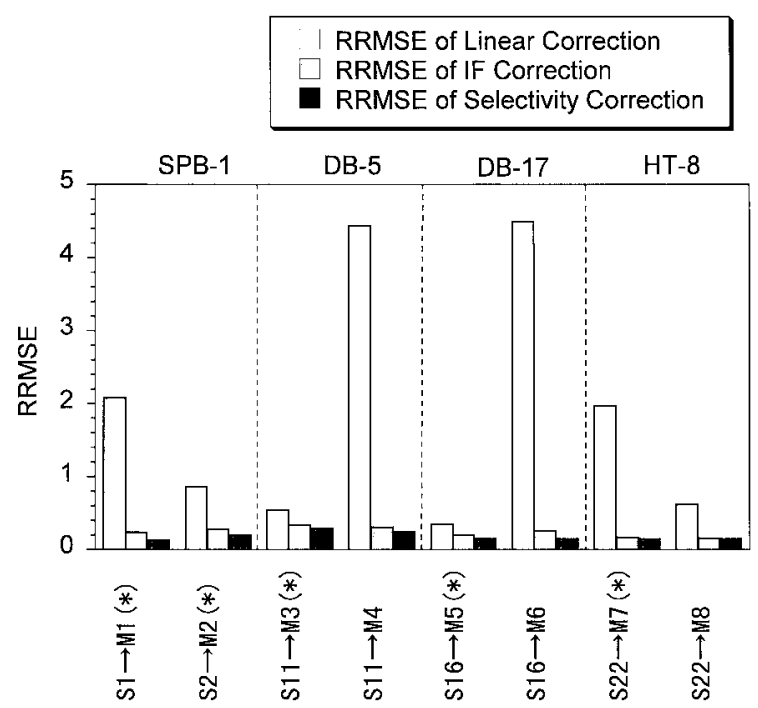

Fig. 5 RRMSEs of the calibrations applied to the measured retention data. "SX $\rightarrow$ MY" indicates that the measured retention data of GC method $\mathrm{Y}$ are calibrated from GC system $\mathrm{X}$ of the retention database. " $*$ " indicates that the temperature program is identical to the reference GC system.

those of the DB-1 columns of the retention database mentioned above. In contrast with the results of the retention database, the column dependency is not significant. In addition, the effects of the selectivity correction are small compared to the results of the retention database. Especially, in the calibration of the HT8 column, the selectivity correction makes a very small improvement. We employed a commercial mixture to measure the retention time of the congeners. In the measured chromatogram, some peaks were heavily overlapped so that errors were included in the measured retention times. The measurement errors may weaken the effects of the selectivity correction. Nevertheless, it is emphasized that the calibration results are comparable to those of the DB-1 columns in the retention database.

The developed calibration method was demonstrated in various GC columns. This method significantly reduced the retention differences between the GC systems with the same stationary phase. The standard errors were within the chromatographic peak widths in most cases. Small prediction errors of the calibration can make very narrow retention

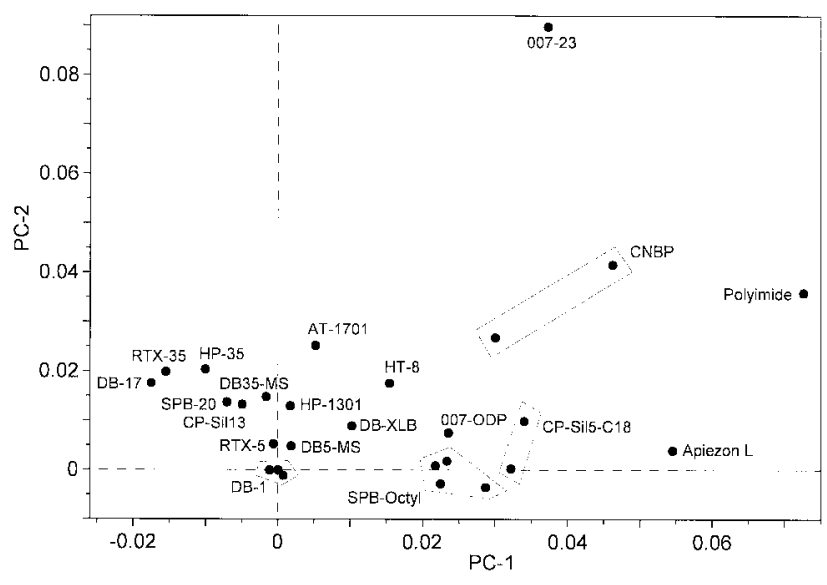

Fig. 6 Score-plot of the first two principal components. The dotted rectangles enclose the GC systems with the same stationary phases.

windows and enable the identification process of the CQCS PCB analysis to be automated by a computer. The residualerror plots are also valuable for validating the congener identification. Large deviations from the calculated retention times expose misidentifications of the PCB congeners.

PCA was applied to selectivity profiles calculated from the retention database. The loading vectors represented dominant patterns of the selectivity profiles and were used to derive the ESPP descriptors. Additionally, the score vectors represent the characteristics of the selectivity profiles. The contribution rates of the first two principal components (PCs) are 61.3\% and $36.2 \%$, respectively, and the accumulated rate is $97.5 \%$. Thus, the first two PCs can sufficiently exhibit the characteristics of the selectivity profiles. The score vectors of the first two PCs are depicted as the score-plot shown in Fig. 6. The score-plot represents the relative selectivity of all GC systems in the retention database. In the score-plot, selectivity differences between GC systems can be measured from the distance between their positions. The shorter is the distance, the more similar is the selectivity. Another criterion is the direction from the origin. ${ }^{23}$ When the GC systems are located with the same direction from the origin, they have similar patterns of selectivity profiles, although the magnitudes of the selectivity coefficients are proportional to the distances from the origin. With these criteria, the score-plot can be used as a guide map for an optimal choice of the stationary phases in dual-column 
PCB analysis. ${ }^{1,21}$ The more dissimilar stationary phases that are used, the greater is the number of congeners that can be separately analyzed.

\section{Acknowledgements}

The authors thank Dr. T. Nakano of Hyogo Prefecture Institute of Environmental Science and Mr. Y. Kammei of JEOL Ltd. for their helpful suggestions.

\section{References}

1. G. Frame, Anal. Chem., 1997, 69, 468A

2. G. M. Frame, Fresenius J. Anal. Chem., 1997, 357, 701

3. H. van den Dool and P. D. Kratz, J. Chromatogr., 1963, 11, 463.

4. G. Castello and G. Testini, J. Chromatogr. A, 1996, 741, 241.

5. S. Chu, X. Miao, and X. Xu, J. Chromatogr. A, 1996, 724 , 392.

6. G. Castello and G. Testini, J. Chromatogr. A, 1997, 787, 251.

7. http://link.springer.de/link/service/journals/00216/index. htm.

8. R. Fischer and K. Ballschmiter, Fresenius Z Anal. Chem., 1988, 332, 441
9. H. Kato and M. Nakata, unpublished.

10. R. Kaliszan, Anal. Chem., 1992, 64, 619A.

11. B. R. Larsen, J. High Resol. Chromatogr., 1995, 18, 141.

12. W. Vetter, B. Luckas, and M. Mohnke, J. Microcolumn Separations, 1996, 8, 183.

13. A. Sakurai, "Spline Functions and Applications", 1981, Tokyo Denki Daigaku Shuppan, Tokyo.

14. K. Sugano, K. Yashimura, and F. Takayama, "Spline Functions by C", ed. A. Sakurai, 1993, Tokyo Denki Daigaku Shuppan, Tokyo.

15. P. J. Green and B. W. Silverman, "Nonparametric Regression and Generalized Linear Models", 1994, Chapman and Hall, London.

16. Y. Miyashita and S. Sasaki, "Chemometrics", 1995, Kyoritsu Shuppan, Tokyo.

17. S. Wold, Chemom. Intell. Lab. Syst., 1987, 2, 37.

18. G. M. Frame, R. E. Wagner, J. C. Carnahan, J. F. Brawn, Jr., R. J. May, L. A. Smullen, and D. L. Bedard, Chemosphere, 1996, 33, 603.

19. G. M. Frame, J. W. Cochran, and S. S. B $\phi$ wadt, J. High Resol. Chromatogr., 1996, 19, 657.

20. G. M. Frame, Fresenius J. Anal. Chem., 1997, 357, 714

21. Y. V. Gankin, A. E. Gorshteyn, and A. Robbat, Jr., Anal. Chem., 1995, 67, 2548.

22. T. Takasuga, T. Inoue, and E. Ohi, J. Environ. Chem., 1995, 5,647

23. O. M. Kvalheim and Y. Liang, Anal. Chem., 1992, 64, 936. 